Archives de sciences sociales des religions L'héritage des Formes

\title{
Le sentiment religieux à l'heure actuelle
}

Émile Durkheim

\section{(2) OpenEdition \\ Journals}

Édition électronique

URL : http://journals.openedition.org/assr/23028

ISSN : 1777-5825

Éditeur

Éditions de l'EHESS

Référence électronique

Émile Durkheim, "Le sentiment religieux à l'heure actuelle », Archives de sciences sociales des religions [En ligne], L'héritage des Formes, mis en ligne le 18 juillet 2011, consulté le 19 avril 2019. URL : http:// journals.openedition.org/assr/23028

(c) Archives de sciences sociales des religions 


\section{Le sentiment religieux à l'heure actuelle}

In: Archives des sciences sociales des religions. N. 27, 1969. pp. 73-78.

Citer ce document / Cite this document :

Durkheim Emile. Le sentiment religieux à l'heure actuelle. In: Archives des sciences sociales des religions. N. 27, 1969. pp. 7378.

doi : 10.3406/assr.1969.2650

http://www.persee.fr/web/revues/home/prescript/article/assr_0003-9659_1969_num_27_1_2650 
Arch. Sociol. des Rel., 27, 1969, 73-77.

Emile DurkheIm

\section{LE SENTIMENT RELIGIEUX}

\section{A L'HEURE ACTUELLE}

ESDAMEs et messieurs, je suis confus de l'honneur qui m'est fait et de l'invitation qui m'est adressée. C'est seulement dans le courant de l'aprèsmidi que M. A., averti que je ne pourrais assister qu'au début de cette séance, est venu me demander de vous adresser quelques paroles. Je vous apporte donc l'expression tout à fait improvisée d'une pensée qui, elle, ne l'est pas mais qui aurait voulu pourtant se présenter à vous sous une forme différente. D'ailleurs je me garderai bien d'anticiper sur le sujet que M. Belot doit traiter ce soir. C'est déjà de sa part une très grande condescendance que de vouloir bien me permettre de prendre la parole avant lui et je l'en remercie. Mais puisqu'il se propose d'examiner devant vous un livre que j'ai récemment publié sur certaines formes de la vie religieuse, je voudrais essayer de vous indiquer très brièvement dans quel esprit je souhaite être étudié et, ensuite, discuté. Et puisque cette assemblée comprend deux sortes d'éléments, libres penseurs d'un côté, libres croyants de l'autre, je vous demanderai la permission de m'adresser à chacun d'eux séparément.

Je m'adresserai d'abord aux libres penseurs, c'est-à-dire aux hommes qui gardent leur entière liberté d'esprit en face de tous les dogmes, même en face de ceux que l'on a quelquefois décorés du beau nom de libre pensée. Pour leur faire comprendre ce qu'il y a d'un peu particulier dans les conceptions que j'ai développées, je leur demanderai de porter surtout leur attention sur une particularité de la vie religieuse qui, sans doute n'est pas ignorée du croyant mais que le libre penseur ne soupçonne pas toujours assez et qui, pourtant, contient la vraie caractéristique de la vie religieuse.

Le plus souvent, les penseurs qui ont entrepris de traduire la religion en termes rationnels n'y ont vu ou n'y ont guère vu qu'un système d'idées, un système de représentations destinées à exprimer telle ou telle portion du réel telle que le sommeil, le rêve, la maladie, la mort ou les grands spectacles de la nature. Or, quand on ne voit dans la religion que des idées ou quand on y voit principalement des idées, il semble vraiment que l'individu ait pu l'édifier par ses seules forces. Sans doute, ces représentations ont quelque chose de déconcertant, elles ont comme un caractère mystérieux qui nous trouble. Mais d'un autre côté, nous savons par expérience que les combinaisons mentales sont si variées, si diverses, si riches, si créatrices qu'a priori nous faisons crédit à l'esprit et nous acceptons volontiers, par avance, que la pensée ait pu, de toutes pièces, inventer ces merveilles. Seule- 
ment et quoique les idéaux religieux aient, par eux-mêmes, des caractères spéciaux, ce n'est pas de ce côté qu'il faut aller chercher ce qu'il y a vraiment de spécifique dans la religion.

La religion, en effet, n'est pas seulement un système d'idées, c'est avant tout un système de forces. L'homme qui vit religieusement, n'est pas seulement un homme qui se représente le monde de telle ou telle manière, qui sait ce que d'autres ignorent ; c'est avant tout un homme qui sent en lui un pouvoir qu'il ne se connaît pas d'ordinaire, qu'il ne sent pas en lui quand il n'est pas à l'état religieux. La vie religieuse implique l'existence de forces très particulières. Je ne puis songer à les décrire ici ; rappelant un mot connu, je me contenterai d'en dire que ce sont ces forces qui soulèvent les montagnes. J'entends par là que, quand l'homme vit de la vie religieuse, il croit participer à une force qui le domine, mais qui, en même temps, le soutient et l'élève au-dessus de lui-même. Appuyé sur elle, il lui semble qu'il peut mieux faire face aux épreuves et aux difficultés de l'existence, qu'il peut même plier la nature à ses desseins.

Ce sentiment-là a été trop général dans l'humanité, il a été trop constant pour qu'il puisse être illusoire. Une illusion ne dure pas ainsi des siècles. Il faut donc que cette force que l'homme sent venir à lui soit réellement existante. Par conséquent, le libre penseur, c'est-à-dire l'homme qui se donne méthodiquement comme tâche d'exprimer la religion par des causes naturelles, sans faire intervenir aucune espèce de notion qui ne soit pas empruntée à nos facultés discursives ordinaires, un tel homme doit se poser la question religieuse dans les termes suivants : de quelle partie du monde de l'expérience peuvent lui venir ces forces qui le dominent et qui, en même temps, le sustentent ?

On comprend très bien que ce n'est pas en essayant d'interpréter tel ou tel phénomène naturel, qu'il nous a été possible de faire venir à nous un pareil afflux de vie. Ce n'est pas d'une représentation erronée du sommeil ou de la mort qu'ont jamais pu surgir des forces de cette nature. Le spectacle des grandes puissances cosmiques ne peut davantage avoir produit cet effet.

C'est là, comme vous le savez peut-être, l'explication rationnelle la plus haute qui ait été proposée de la religion. Mais les forces physiques ne sont que des forces physiques ; par conséquent, elles restent en-dehors de moi. Je puis les voir du dehors; elles ne me pénètrent pas, elles ne viennent pas se mêler à ma vie intérieure. Je ne me sens pas plus fort, mieux armé contre les destinées, moins asservi à la nature parce que je vois les fleuves couler, les moissons germer, les astres accomplir leurs révolutions : il n'y a que des forces morales que je puisse sentir en moi, qui puissent me commander et me réconforter. Et encore une fois, il faut que ces forces soient réelles, qu'elles soient réellement en moi. Car ce sentiment de réconfort et de dépendance n'est pas illusoire.

Ainsi déterminé, le problème se pose done dans des termes assez simples. Pour expliquer la religion, pour la rendre rationnellement intelligible - et c'est ce que propose le libre penseur - il faut trouver dans le monde que nous pouvons atteindre par l'observation, par nos facultés humaines, une source d'énergies supérieures à celles dont dispose l'individu, et qui pourtant puissent se communiquer à lui. Or je demande si cette source peut être trouvée ailleurs que dans cette vie très particulière qui se dégage des hommes assemblés. Nous savons en effet par expérience que, quand les hommes sont réunis, quand ils vivent d'une vie commune, de leur réunion même surgissent des forces exceptionnellement intenses qui les dominent, les exaltent, portent leur ton vital à un degré qu'ils ne connaissent pas dans la vie privée. Sous l'effet de l'entraînement collectif, ils sont 
parfois saisis d'un véritable délire qui les pousse à des actes où ils ne se reconnaissent pas eux-mêmes.

Je ne puis songer à exposer ici, même succinctement, les analyses et les faits sur lesquels j'ai appuyé cette thèse fondamentale. Je me bornerai seulement à avertir ceux de mes auditeurs qui ne m'ont pas lu, que cette manière d'entendre et d'expliquer la religion, si elle ne se présente pas comme une vérité démontrée, ne repose pas cependant sur des vues purement dialectiques. Ce n'est pas une hypothèse abstraite et purement philosophique. Mais, dégagée des faits et de l'observation historique, elle a déjà inspiré plus d'une recherche particulière qu'elle a guidée utilement; déjà elle a servi à interpréter, dans des religions diverses, des phénomènes divers; elle a donc subi l'épreuve de l'expérience et prouvé ainsi sa vitalité.

Mais je m'abstiens d'insister sur les raisons qui militent en faveur de la conception qui vous sera exposée tout à l'heure en toute impartialité, j'en suis sûr, et discutée avec une égale liberté. L'unique objet que je me propose est de vous préparer à écouter cet exposé et cet examen ; il ne m'appartient pas de l'anticiper. En résumé, ce que je demande au libre penseur, c'est de se placer en face de la religion dans l'état d'esprit du croyant. C'est à cette condition seulement qu'il peut espérer la comprendre. Qu'il la sente telle que le croyant la sent, car elle n'est véritablement que ce qu'elle est pour ce dernier. Aussi quiconque n'apporte pas à l'étude de la religion une sorte de sentiment religieux ne peut en parler! Il ressemblerait à un aveugle qui parlerait des couleurs. Or, pour le croyant, ce qui constitue essentiellement la religion, ce n'est pas une hypothèse plausible ou séduisante sur l'homme ou sur sa destinée; ce qui l'attache à sa foi, c'est qu'elle fait partie de son être, c'est qu'il ne peut y renoncer, lui semble-t-il, sans perdre quelque chose de lui-même, sans qu'il en résulte une dépression, une diminution de sa vitalité, comme un abaissement de sa température morale.

En un mot, la caractéristique de la religion, c'est l'influence dynamogénique qu'elle exerce sur les consciences. Expliquer la religion, c'est donc avant tout, expliquer cette influence.

Je m'adresse maintenant au libre croyant, à l'homme qui, tout en ayant une religion, tout en adhérant même à une formule confessionnelle, apporte cependant à l'examen de cette formule une liberté d'esprit qu'il s'efforce de rendre aussi entière que possible, et je lui tiendrai un autre langage.

Je lui demanderai sa sympathie. Je crois que la conception que je m'efforce d'accréditer mérite cette sympathie. Certes, si l'on tient à une formule confessionnelle d'une manière exclusive et intraitable, si l'on croit que l'on possède la vérité religieuse sous sa forme définitive, alors l'entente est impossible et ma présence ici n'aurait aucun sens. Mais si l'on estime que les formules ne sont que des expressions provisoires, qui ne durent et ne peuvent durer qu'un temps, si l'on pense qu'elles sont toutes imparfaites, que l'essentiel, ce n'est pas la lettre de ces formules mais la réalité qu'elles recouvrent et qu'elles expriment toutes plus ou moins inexactement, qu'il faut, par conséquent, dépasser la surface pour atteindre la force des choses, alors, je crois qu'il y a une entreprise que nous pouvons poursuivre d'un commun accord, au moins jusqu'à un certain point.

Mais il faut y apporter cette liberté d'esprit ; il faut, pour un temps, pratiquer une sorte de doute cartésien. Sans aller jusqu'à suspecter la formule à laquelle on croit, on doit tout au moins l'oublier provisoirement sauf à y revenir plus tard. Une fois qu'on s'est soustrait à cette tyrannie, on n'est plus exposé à commettre 
l'erreur et l'injustice dans laquelle sont tombés certains croyants qui ont qualifié de foncièrement irréligieuse ma façon d'interpréter la religion.

Il ne peut pas y avoir une interprétation rationnelle de la religion qui soit foncièrement irréligieuse, une interprétation irréligieuse de la religion serait une interprétation qui nierait le fait dont il s'agit de rendre compte. (Applaudissements). Rien n'est plus contraire à la méthode scientifique. Ce fait, nous pouvons le comprendre différemment, nous pouvons même arriver à ne pas le comprendre, mais nous ne saurions le nier.

Et en effet, quand on se refuse à confondre la religion avec tel ou tel dogme particulier, ce qu'on voit surtout en elle, e'est un ensemble d'idéaux qui ont pour effet d'élever l'homme au-dessus de lui-même, de l'amener à se déprendre de ses intérêts temporels et vulgaires et de lui faire vivre une existence qui dépasse en valeur et en dignité celle qu'il mène quand il ne s'occupe que d'assurer sa subsistance. Or ce qu'implique la doctrine dont il va vous être parlé et dont j'ai rappelé tout à l'heure les lignes essentielles, c'est que, par-dessus tous les dogmes et toutes les confessions, il existe une source de vie religieuse, aussi vieille que l'humanité et qui ne peut jamais se tarir : c'est celle qui résulte de la fusion des consciences, de leur communion dans une même pensée, de leur coopération à une même œuvre, de l'action moralement tonifiante et stimulante que toute communauté d'hommes exerce sur ses membres. N'y a-t-il pas là une proposition sur laquelle nous pouvons tomber d'accord ? Sans doute, vous pouvez penser que cette vie religieuse n'est pas suffisante, qu'il en est une, plus haute, qui dérive d'une tout autre origine. Mais n'est-ce pas quelque chose que de pouvoir reconnaître qu'il existe en nous, hors de nous, des forces religieuses qu'il dépend de nous de dégager, d'appeler à l'existence, que nous ne pouvons même pas ne pas engendrer par cela seul que nous nous rapprochons les uns des autres, que nous pensons, sentons, agissons en commun.

Naguère, un orateur, nous montrant les cieux d'un geste prophétique, nous disait qu'ils se vident et nous engageait à tourner nos regards vers la terre, c'est-àdire à nous occuper avant tout d'aménager le mieux possible nos intérêts économiques. On a dit de la formule qu'elle est impie. Du point de vue auquel je me place, on peut dire qu'elle est fausse. Non, il n'est pas à craindre que jamais les cieux se dépeuplent d'une manière définitive; car c'est nous-mêmes qui les peuplons. Ce que nous y projetons, ce sont des images agrandies de nous-mêmes. Et tant qu'il y aura des sociétés humaines, elles tireront de leur sein de grands idéaux dont les hommes se feront les serviteurs.

Dans ces conditions, n'est-il pas permis de dire qu'une conception sociale de la religion est nécessairement animée d'un souffle religieux qu'on ne peut méconnaître sans injustice?

Mais pour préciser ces idées, on voudrait se représenter un peu en quoi pourra consister une religion de l'avenir, c'est-à-dire une religion plus consciente de ses origines sociales. Certes, on ne saurait s'exprimer sur ce point avec trop de réserve. Il est tout à fait vain de chercher à deviner sous quelle forme précise une telle religion arrivera à s'exprimer. Mais ce qu'on peut entrevoir, ce sont les forces sociales qui l'engendreront.

Si aujourd'hui notre vie religieuse se languit, si les renaissances passagères qu'on signale ne font jamais l'effet que de mouvements superficiels et passagers, ce n'est pas parce qu'on s'est détourné de telle ou telle formule confessionnelle, mais c'est que notre puissance créatrice d'idéaux a faibli. Mais c'est que nos sociétés traversent une phase d'ébranlement profond. De cet ébranlement qu'elles 
subissent, elles peuvent être fières, en un sens ; car il vient de ce que, ayant passé la période d'équilibre où elles pouvaient vivre tranquillement du passé, elles sont obligées de se renouveler et se cherchent laborieusement, douloureusement. Les vieux idéaux et les divinités qui les incarnaient sont en train de mourir parce qu'ils ne répondent plus suffisamment aux aspirations nouvelles qui se sont fait jour, et les nouveaux idéaux qui nous seraient nécessaires pour orienter notre vie ne sont pas nés. Nous nous trouvons ainsi dans une période intermédiaire, période de froid moral qui explique les manifestations diverses dont nous sommes, à chaque instant, les témoins inquiets ou attristés.

Mais qui ne sent - et c'est ce qui doit nous rassurer - qui ne sent que, dans les profondeurs de la société, une vie intense s'élabore, qui cherche ses voies d'issue et qui finira bien par les trouver. Nous aspirons à une justice plus haute qu'aucune des formules existantes n'exprime de manière à nous satisfaire. Mais ces aspirations obscures qui nous travaillent, arriveront, un jour ou l'autre, à prendre plus clairement conscience d'elles-mêmes, à se traduire en des formules définies autour desquelles les hommes se rallieront et qui deviendront un centre de cristallisation pour des croyances nouvelles. Quant à la lettre de ces croyances, c'est ce qu'il est inutile de chercher à percevoir. Resteront-elles générales et abstraites, se rattacheront-elles à des êtres personnels qui les incarneront et les représenteront? Ce sont là contingences historiques que l'on ne saurait prévoir.

Tout ce qu'il importe, c'est de sentir, par-dessous le froid moral qui règne à la surface de notre vie collective, les sources de chaleur que nos sociétés portent en elles-mêmes. On peut même aller plus loin et dire avec quelque précision dans quelle région de la société ces forces neuves sont particulièrement en voie de formation : c'est dans les classes populaires.

M. et M., il y a une idée à laquelle il faut nous faire de toute nécessité : c'est que l'humanité est abandonnée, sur cette terre, à ses seules forces et ne peut compter que sur elle-même pour diriger ses destinées. A mesure qu'on avance dans l'histoire, cette idée n'a fait que gagner du terrain; je doute donc qu'elle en perde dans l'avenir. Au premier abord, elle peut troubler l'homme qui est habitué à se représenter comme extra-humaines les forces sur lesquelles il s'appuie. Mais s'il arrive à se convaincre que l'humanité elle-même peut lui fournir cet appui dont il a besoin, n'y a-t-il pas dans cette perspective quelque chose de hautement réconfortant, puisque les ressources qu'il réclame se trouvent ainsi mises à sa portée et, pour ainsi dire, sous sa main ? Or c'est à cette conception qu'aboutit la théorie dont M. Belot veut bien vous entretenir.

Emile Durkherm 
